jz-2021-03671p.R1

Name: Peer Review Information for "Photo-Charging Effect on Catalysis of Metallic Nanoparticles"

First Round of Reviewer Comments

Reviewer: 1

\title{
Comments to the Author
}

Lyu et al. charge colloidal Pd nanoparticles by photoexcitation and then use the charged particles to catalyze the reduction of 4-nitrophenol by sodium borohydride in the dark. The work appears comprehensive aside for a potential flaw in the quantitative estimation of accumulated charge concentration. However, the concept itself is based on past work and entirely expected; so the paper falls short on 'new physical insights'. I recommend that the points below be addressed and a full-length article containing contrast and comparisons with past work on photocharging and catalysis by photocharged nanoparticles be submitted to the Journal of Physical Chemistry.

1) Lack of new physical insights or conceptual advance:

a) The opening statement "Photo-charging effect is widely known across different research fields, but rarely studied for catalysis" is not representative of the status of the field. Multiple groups: Mulvaney, Jain, and W. David Wei have described catalysis by photocharged metal nanoparticles in papers spanning more than a decade. It is one of the mechanisms invoked in plasmonic catalysis. For instance,

a) Mulvaney and coworkers in a 2008 paper (10.1038/nnano.2008.246) charged nanoparticles using reducing agents, measured the charge on a nanoparticle, and used these charged nanoparticles to catalyze redox reactions.

b) Jain and coworkers in a series of papers showed photocharging of metal nanoparticles by light excitation, both plasmonic and non-plasmonic, and used these photocharged nanoparticles for the catalysis of $\mathrm{CO} 2$ reduction (10.1021/acs.nanolett.7b05410), multielectron redox chemistry (10.1038/s41557-018-0054-3) and hydrogen evolution reaction (10.1021/acs.jpcc.9b10473). The strategy employed in the current paper of using an alcohol hole scavenger along with photoexcitation appears to be derived from the first two works (10.1038/s41557-018-0054-3 and 10.1021/acs.nanolett.7b05410) without proper attribution. The catalytic activity was shown to be a function of the degree of photocharging in pat work (10.1557/mrs.2019.293). Past work also showed 
that the degree of photocharging is directly dependent on the light intensity (10.1002/anie.201914118) and also tunable by the excitation wavelength (10.1038/s41557-018-0054-3 and

10.1021/acs.jpcc.9b10473). The authors' findings are in line with what is known from this past work. Furthermore, it has been shown that the degree of photocharging influences the selectivity in $\mathrm{CO} 2$ reduction with higher accumulated charge densities favoring $\mathrm{C} 2$ and $\mathrm{C} 3$ products over a $\mathrm{C} 1$ product (10.1021/acs.nanolett.7b05410 and 10.1038/s41467-019-10084-5).

c) W. David Wei and coworkers have demonstrated (10.1038/nmat4683) the use of photocharging of metal nanoparticles to catalyze their growth into nanoprisms.

The submission by Lyu et al. appears to avoid citing the work listed in a-c above. This ought to be remedied. The paper could be made scholarly by presenting an accurate description of the current state of knowledge in the field as represented by the body of published work. The abstract, introduction, and discussion sections ought to be rewritten with this context and the authors' findings compared and contrasted with this past work in the field. Such a revision would be necessary for the the paper to be accurately representative of the field and meaningful to readers of the journal.

d) The finding "As the power increased, each nanoparticle had an averaged increase in absorbed photons and generated carriers." has been made quantitatively in the past (see

10.1021/acs.nanolett.6b01373 and 10.1038/s41557-018-0054-3) and ought to be discussed, compared, and contrasted in this context. Without such a discussion, it is difficult to gauge if the findings represent new physical insights.

e) The effect of the oxidation potential of the hole scavenger and of the excitation wavelength on the catalytic rate, described in Fig. 4, have been observed in a past study as well (10.1038/s41557-018-00543 and 10.1021/acs.jpcc.9b10473) and ought to be discussed, compared, and contrasted in this context. Without such a discussion, it is difficult to gauge if the findings represent new physical insights.

2) Need for scholarly citation practices:

The paper needs considerable improvement on scholarly practices of citation and attribution of ideas.

a) The statement: "During a photocatalyzed reaction, when there is an imbalance in consuming either electrons or holes on metallic nanoparticle catalysts, the consequent photo-charging may create an extra catalytic process besides the well-known hot-carrier or plasmon mediated catalysis" is presented without citations or support. This idea is introduced in the papers starting in 2016 (see some examples 
in point $1 \mathrm{~b}$ above) and recently articulated in an account of this body of work (10.1021/acs.accounts.0c00378).

b) The statement "This extra process acts as a hidden background, which is often overlooked" is not a true reflection of the current status of the field because photocharging has been studied and exploited for plasmonic catalysis in a body of work. Yes, photocharging is less commonly invoked as a mechanism than hot electrons; but it has not been overlooked and readers ought to be pointed to the existing body of work rather than attempting to cast aside this past precedent.

c) The description starting with "...photocharging effect is usually demonstrated via composite systems such as Ag-TiO2,2 Au-TiO2,10 or WO3-TiO2 11, where one material absorbs light and generates hot carriers while the other creates a barrier for charge separation and stores the charge for further reactions..." gives the impression that metal nanoparticles have not by themselves used for studies of photocharging and photocharging-induced catalysis, which is not an accurate reflection of the field. This statement ought to be corrected and the work on metal nanoparticles (without oxides) ought to be introduced with citations to examples.

d) "In principle, metallic nanoparticles can undergo photo-charging when the photogenerated hot electrons (or hot holes) are quenched by sacrificial agents, leaving ground-state holes (or electrons) on the particles.19, 20" The references are review articles and do not represent the primary source/s where this concept was described. Some examples include 10.1021/acs.nanolett.6b01373, 10.1021/acs.nanolett.7b05410, and 10.1038/s41557-018-0054-3. Once again, the paper could be made scholarly by proper attribution of ideas from the parent source.

\section{3) Technical questions}

a) From the procedure it appears that the reaction mixture is added to the photocharged Pd nanoparticle solution immediately after the light excitation is turned off. It is likely that the "just photocharged" Pd nanoparticle solution has an elevated temperature than the ambient medium due to the effect of photothermal heating. Was this temperature measured for each of the photoexcitation conditions? The control experiments with uncharged Pd nanoparticles + IPA ought to be performed at these temperatures to account for the potential impact of photothermal enhancement of catalytic activity.

b) There is a potentially serious flaw in the titration method for determination of accumulated electron concentration. Under constant photoexcitation, there is a steady-state achieved between the rate of generation of electron-hole pairs on one hand and the rate of electron-hole annihilation (by charge 
transfer or recombination) on the other (10.1038/s41557-018-0054-3). When the photoexcitation is turned off, electron-hole pair production stops but the excess electrons continue to be removed by reactions with protons in the medium. The accumulated electrons do not stay on the nanoparticle indefinitely. The molar amount of thionine reduced therefore would not be a direct measure of the number of electrons extracted from the nanoparticle but would rather be a reflection of the ratio of the rate of electron transfer to thionine to that of rate of electron consumption by capture by protons. One can build an analytical model to relate these rates to the accumulated electron concentration on the nanoparticles; but treating a dynamically evolving system using a titration approach appears to be flawed. The resulting numbers are unlikely to be quantitatively representative.

c) The accumulated electron concentration is found to be nearly the same in nanoparticles exposed to $153 \mathrm{~mW}$ of $405 \mathrm{~nm}$ light and those that were not exposed to light (see Fig. 2d). This finding may also reflect flaw/s in the thionine titration method for determination of accumulated electron concentration.

d) As Figure S2 shows, the nanoparticle sample has severe heterogeneity. How does this affect the estimation of accumulated electron concentrations and batch-to-batch variations in catalytic activity? These points ought to be discussed in the main text. Statistics of nanoparticle size and shape ought to be provided as well.

e) If there is significant electronic charge on Pd nanoparticles resulting simply from the use of ascorbic acid in the synthesis, as stated in the supporting information (see page S8), then the entire study ought to be re-analyzed. The effect of this residual charge and that formed by photoexcitation on the catalytic activity will need to be separated.

Reviewer: 2

Comments to the Author

In this manuscript, the authors reported photo-charging effect on catalysis of metallic nanoparticles. The obtained results are of some interest. However, the manuscript needs some minor revisions by considering the following points.

1. Did the charging time effect the Pd nanoparticle size? The catalytic activity of the particles dropped after a longer charging time may also be caused by the change in particle size.

2. Except as a reducing agent, $\mathrm{NaBH} 4$ is also regarded as a promising hydrogen storage material, which should be introduced in the INTRODUCTION of this manuscript. Some references (such as Inorg. Chem. Front., 2021, 8,1056-1065; Inorg. Chem. Front., 2020, 7,3837-3874) can be cited.

3. There are a few typing errors spotted. The authors need to proof read the manuscript. 
Reviewer: 3

\section{Comments to the Author}

This work by Pin Lyu and Son C. Nguyen reports the photo-charging effect on catalysis of metallic nanoparticles. The mechanism underlying a proportional correlation between accumulated electrons and catalytic improvement of Pd nanoparticles was proposed and was well-supported by the control experiment. This work is of considerable interest, and I recommend its publication in JPCL upon addressing my concerns as listed below:

More detailed comments:

(1) The authors reported the use of IPA as a class of hole scavengers; how to determine the degree of hole extraction in the experimental conditions?

(2) With longer wavelength light as an excitation source (470 or $530 \mathrm{~nm}$ ), the photo-charging effect was not strong (Figure $3 \mathrm{c}$ ). How to explain this phenomenon in detail?

(3) For the catalysis step, the authors chose the reduction of 4-nitrophenol by $\mathrm{NaBH} 4$ as a model that demonstrated accumulated charges resulting in catalytic improvement of the photo-charged nanoparticles. Does this conclusion apply to other models?

(4) Can the density of electrons on the surface Pd nanoparticles reach a saturated state when exciting the nanoparticles for a long time?

Author's Response to Peer Review Comments:

We would like to thank the editor and reviewers for their constructive comments, which help to greatly improve our manuscript greatly. All reviewers' comments are copied without any omission, and our point-by-point responses are colored. Accordingly, we have attached the clean version of the revised manuscript, and the annotated version with highlighted change.

Non-scientific changes:

1) Supporting Information Statement: Please provide a brief description of the contents of the supplementary material at the end of the manuscript file before the references under the heading "Supporting Information". A brief, nonsentence description of the actual contents of each file is required. This description should be labeled Supporting Information and should appear before the Acknowledgement and Reference sections. Examples of sufficient and insufficient descriptions are as follows: 
*Examples of sufficient descriptions: "Supporting Information: 1H NMR spectra for all compounds" or "Additional experimental details, materials, and methods, including photographs of experimental setup".

*Examples of insufficient descriptions: "Supporting Information: Figures S1-S3" or "Additional figures as mentioned in the text".

Response: The Supporting Information Statement was revised as requested.

Supporting Information. Experimental section including synthesis of Pd nanoparticles, photocharging procedures, reaction conditions and estimation of stored electrons. and Supporting figures, tables, and notes are included.Figures S1-S5 for Pd nanoparticle characterization, spectral evolution of reaction solution, and XPS analysis. Tables S1-S2 for photon fluxes. Note S1 for estimating number of stored electrons and quantum yield of photo-charging.

2) References: We noticed that reference 18 is "under review." Please update the status of this reference. (References must be published in order to be included in the manuscript.)

Response: Unfortunately, reference 18 (now as 23 in the revised manuscript) is still under review. We submitted the manuscript of this reference to ChemRxiv and updated its DOI (10.26434/chemrxiv-20212qx43) accordingly.

3) TOC Graphic: Please change the TOC header from "TOC Graphics" to "TOC Graphic". Response: It was revised as requested.

4) References: In both the main file and the supporting information, fix the style of all references to use $J P C L$ formatting (check all references carefully). ${ }^{* * * J P C}$ Letters reference formatting requires that journal references should contain: () around numbers, author names, article title (titles entirely in title case or entirely in lower case), abbreviated journal title (italicized), year (bolded), volume (italicized), and pages (first-last). Book references should contain author names, book title (in the same pattern), publisher, city, and year.

Response: All references were carefully checked and updated as requested. 
Reviewer(s)' Comments to Author:

\section{Reviewer: 1}

Recommendation: Reconsider as an article in The Journal of Physical Chemistry A/B/C.

\section{Comments:}

Lyu et al. charge colloidal Pd nanoparticles by photoexcitation and then use the charged particles to catalyze the reduction of 4-nitrophenol by sodium borohydride in the dark. The work appears comprehensive aside for a potential flaw in the quantitative estimation of accumulated charge concentration. However, the concept itself is based on past work and entirely expected; so the paper falls short on 'new physical insights'. I recommend that the points below be addressed and a full-length article containing contrast and comparisons with past work on photocharging and catalysis by photocharged nanoparticles be submitted to the Journal of Physical Chemistry.

Response: We would like to thank the reviewer for the constructive comments. The new physical insight of this work is the photo-charging step was separated from photocatalysis step, and the catalysis of the photo-charged metallic nanoparticles was evaluated under non-irradiation condition. This condition allowed us to evaluate the catalytic contribution of photo-charging when the photocatalysts are not absorbing photons. We would like to remind the readers of this background catalysis when studying photocatalysis. Previous novel papers (mostly published by Jain and coworkers) mentioned by the reviewer have beautifully demonstrated the versatile application as well as the catalytic mechanism of photo-charging and photocatalysis of photo-charged nanoparticles. However, those papers did not demonstrate the catalysis of photo-charged nanoparticles under the dark condition. Our work implies that photo-charged nanoparticles can still catalyze a reaction even when they no longer absorb new photons, or they are not undergoing photoexcitation and awaiting for absorbing new photons.

We now add the below text in the abstract and introduction to emphasize our new physical insight.

"This work reminds us that photo-charged nanoparticles may still catalyze chemical reactions as a background phenomenon even when they are not undergoing photoexcitation."

"Moreover, previous studies have explored the photocatalysis of photo-charged metallic nanoparticles under continuous irradiation. It is important to see if the photo-charged metallic nanoparticles can still catalyze chemical reactions after stopping irradiation."

1) Lack of new physical insights or conceptual advance:

a) The opening statement "Photo-charging effect is widely known across different research fields, but rarely studied for catalysis" is not representative of the status of the field. Multiple groups: Mulvaney, Jain, and W. David Wei have described catalysis by photocharged metal nanoparticles in papers spanning more than a decade. It is one of the mechanisms invoked in plasmonic catalysis. For instance,

Response: We meant photo-charging is rarely studied for all kinds of catalysis, and we did not mean this statement is only limited to photocatalysis. We made the below update in the abstract to narrow the scope of this work to photocatalysis:

"Photo-charging effect is widely known across different research fields, but rarely studied for quantified as a background contribution in photocatalysis,"

We did cite the work from the two representative groups, the Brus from Columbia University and the Jain from the University of Illinois Urbana-Champaign. We cited both of their very first work as well as their Acc. Chem. Res. papers which already reviewed all the papers that the reviewer mentioned. 
Mulvaney's work does not fall into photochemistry category, and we added W. David Wei's work in the revision.

a) Mulvaney and coworkers in a 2008 paper (10.1038/nnano.2008.246) charged nanoparticles using reducing agents, measured the charge on a nanoparticle, and used these charged nanoparticles to catalyze redox reactions.

Response: We would like to point out that Mulvaney and coworker did not use photo-charging approach in their 2008 Nat. Nanotechnol. paper. Gold nanoparticles were charged by electron injection from ascorbic acid. The light source was used to detect spectral shift of plasmon resonance, but photocharging was never considered in the manuscript. This is the reason why this paper is not a representative work for photo-charging induced catalysis. On a side note, the authors had not established a relationship between the amount of charge and the catalytic activity.

b) Jain and coworkers in a series of papers showed photocharging of metal nanoparticles by light excitation, both plasmonic and non-plasmonic, and used these photocharged nanoparticles for the catalysis of $\mathrm{CO} 2$ reduction (10.1021/acs.nanolett.7b05410), multielectron redox chemistry (10.1038/s41557-018-0054-3) and hydrogen evolution reaction (10.1021/acs.jpcc.9b10473). The strategy employed in the current paper of using an alcohol hole scavenger along with photoexcitation appears to be derived from the first two works (10.1038/s41557-018-0054-3 and 10.1021/acs.nanolett.7b05410) without proper attribution. The catalytic activity was shown to be a function of the degree of photocharging in pat work (10.1557/mrs.2019.293). Past work also showed that the degree of photocharging is directly dependent on the light intensity (10.1002/anie.201914118) and also tunable by the excitation wavelength (10.1038/s41557-018-0054-3 and 10.1021/acs.jpcc.9b10473). The authors' findings are in line with what is known from this past work. Furthermore, it has been shown that the degree of photocharging influences the selectivity in $\mathrm{CO} 2$ reduction with higher accumulated charge densities favoring $\mathrm{C} 2$ and $\mathrm{C} 3$ products over a $\mathrm{C} 1$ product (10.1021/acs.nanolett.7b05410 and 10.1038/s41467-019-10084-5).

Response: We would like to clarify that we did cite Jain and coworkers' work in the introduction. Ref 16 in our previous version is their very first publication on photo-charging. We then cited their Acc. Chem. Res paper (Ref 8 in our previous version), which already has all the papers that the reviewer mentioned.

We think this comment will help the readers find more applications of photo-charging in photocatalysis, thus we added more citations as suggested. The following text was added to the introduction:

"Recent studies have a similar approach to create light-induced voltages for lowering the activation energy barrier of chemical reactions, ${ }^{8,16}$ catalyze anisotropic growth of gold nanoprisms, ${ }^{17}$ control multi-electron transfer for better catalytic selectivities, ${ }^{18,19}$ enhance photochemical potential for redox reactions, ${ }^{20,21}$ improve catalytic activities under different excitation wavelengths ${ }^{14,22,23}$ or tune reduction potential of nanoparticle photocatalysts 9 ."

c) W. David Wei and coworkers have demonstrated (10.1038/nmat4683) the use of photocharging of metal nanoparticles to catalyze their growth into nanoprisms.

Response: We did not cite this elegant work because the authors did not focus on catalysis. We thank the reviewer for reminding us, and we added this work in the introduction of the revised version.

The submission by Lyu et al. appears to avoid citing the work listed in a-c above. This ought to be remedied. The paper could be made scholarly by presenting an accurate description of the current state of knowledge in the field as represented by the body of published work. The abstract, introduction, and discussion sections ought to be rewritten with this context and the authors' findings compared and 
contrasted with this past work in the field. Such a revision would be necessary for the the paper to be accurately representative of the field and meaningful to readers of the journal.

Response: As we clarified above, we cited the review and representative papers. Besides, our intention is not about highlighting the applications of photo-charging in photocatalysis. Our intention is introducing some representative work and focusing on the background catalysis of photo-charged nanoparticles when these particles are not undergoing photoexcitation. We hope the revised version makes our points clearer.

d) The finding "As the power increased, each nanoparticle had an averaged increase in absorbed photons and generated carriers." has been made quantitatively in the past (see 10.1021/acs.nanolett.6b01373 and 10.1038/s41557-018-0054-3) and ought to be discussed, compared, and contrasted in this context. Without such a discussion, it is difficult to gauge if the findings represent new physical insights.

Response: The statement "As the power increased, each nanoparticle had an averaged increase in absorbed photons and generated carriers" is generally true and it does not need citations. We now cited the 10.1021/acs.nanolett.6b01373 paper on page S8 in the SI and briefly compared the numbers of accumulated electrons.

"up to around 8800 electrons per photo-charged gold nanoparticles $(13 \mathrm{~nm}),{ }^{15}$ ",

We however want to clarify that our experiment condition is very different from conditions in these two papers, thus the suggested comparison is not straightforward. We used porous Pd nanoparticles, and we separated photo-charging step from catalysis step. We added the below text on page 8 of the main text to show our experiments have the same trend with previous studies.

"Jain and coworkers reported an increase in reduction rate of ferricyanide catalyzed by gold nanoparticle photocatalysts after increasing photon flux. ${ }^{16,19}$ Despite the different experimental conditions, mainly due to our separation of photo-charging and catalyzing reaction, our results show a similar trend. While multi-electron transfer reactions were possible under continuous irradiation in previous studies, ${ }^{14,17,19}$ the observation of 4-aminophenol product (a rising peak at $300 \mathrm{~nm}$ in Figure 1) in this study indicates a six-electron reduction was achieved under our photocharging, then stopping irradiation and evaluating catalysis."

e) The effect of the oxidation potential of the hole scavenger and of the excitation wavelength on the catalytic rate, described in Fig. 4, have been observed in a past study as well (10.1038/s41557-018-00543 and 10.1021/acs.jpcc.9b10473) and ought to be discussed, compared, and contrasted in this context. Without such a discussion, it is difficult to gauge if the findings represent new physical insights.

Response: We thank the reviewer for this important suggestion. We added the below text on pages 12 and 13 to compare to previous work.

"Similar wavelength-dependent trend was also observed for gold nanoparticle photocatalysts under continuous irradiation. ${ }^{14,19,20,}$

"Similarly, IPA and EtOH scavengers do not give a drastic difference in rate constants of ferricyanide reduction catalyzed by gold nanoparticle photocatalysts under continuous irradiation. ${ }^{19}$ ",

2) Need for scholarly citation practices:

The paper needs considerable improvement on scholarly practices of citation and attribution of ideas. 
a) The statement: "During a photocatalyzed reaction, when there is an imbalance in consuming either electrons or holes on metallic nanoparticle catalysts, the consequent photo-charging may create an extra catalytic process besides the well-known hot-carrier or plasmon mediated catalysis" is presented without citations or support. This idea is introduced in the papers starting in 2016 (see some examples in point $1 \mathrm{~b}$ above) and recently articulated in an account of this body of work (10.1021/acs.accounts.0c00378).

Response: Our statement is generally true, hence we thought adding a citation was not necessary. We now cited the 10.1021/acs.accounts.0c00378 paper as suggested.

b) The statement "This extra process acts as a hidden background, which is often overlooked" is not a true reflection of the current status of the field because photocharging has been studied and exploited for plasmonic catalysis in a body of work. Yes, photocharging is less commonly invoked as a mechanism than hot electrons; but it has not been overlooked and readers ought to be pointed to the existing body of work rather than attempting to cast aside this past precedent.

Response: Following the context of our paragraph, catalysis of photo-charged particles was evaluated under dark condition. Thus, we consider this extra catalytic process exists besides the well-known hotcarrier or plasmon mediated catalysis. All the work the reviewer mentioned was based on photoexcitation of photo-charged nanoparticles and the evaluation of their photocatalysis. We stop irradiation of our photo-charged nanoparticles and we evaluate their catalysis at their ground state.

c) The description starting with "...photocharging effect is usually demonstrated via composite systems such as Ag-TiO2,2 Au-TiO2,10 or WO3-TiO2 11, where one material absorbs light and generates hot carriers while the other creates a barrier for charge separation and stores the charge for further reactions..." gives the impression that metal nanoparticles have not by themselves used for studies of photocharging and photocharging-induced catalysis, which is not an accurate reflection of the field. This statement ought to be corrected and the work on metal nanoparticles (without oxides) ought to be introduced with citations to examples.

Response: We thank reviewer for this correction. We revised the first paragraph accordingly. We now cited Brus and Jain work in the introduction.

d) "In principle, metallic nanoparticles can undergo photo-charging when the photogenerated hot electrons (or hot holes) are quenched by sacrificial agents, leaving ground-state holes (or electrons) on the particles.19, 20" The references are review articles and do not represent the primary source/s where this concept was described. Some examples include 10.1021/acs.nanolett.6b01373, 10.1021/acs.nanolett.7b05410, and 10.1038/s41557-018-0054-3. Once again, the paper could be made scholarly by proper attribution of ideas from the parent source.

Response: We cited those suggested papers in the revised version.

3) Technical questions

a) From the procedure it appears that the reaction mixture is added to the photocharged Pd nanoparticle solution immediately after the light excitation is turned off. It is likely that the "just photocharged" Pd nanoparticle solution has an elevated temperature than the ambient medium due to the effect of photothermal heating. Was this temperature measured for each of the photoexcitation conditions? The control experiments with uncharged Pd nanoparticles + IPA ought to be performed at these temperatures to account for the potential impact of photothermal enhancement of catalytic activity. 
Response: In our experiment, the fan and cuvette holder were used to cool the photo-charged Pd nanoparticle solution to room temperature. We added the below text to the main text to address this concern:

"The overall temperature of photo-charged solutions rose only $1-2{ }^{\circ} \mathrm{C}$, which had almost no influence on the catalyzed reactions."

b) There is a potentially serious flaw in the titration method for determination of accumulated electron concentration. Under constant photoexcitation, there is a steady-state achieved between the rate of generation of electron-hole pairs on one hand and the rate of electron-hole annihilation (by charge transfer or recombination) on the other (10.1038/s41557-018-0054-3). When the photoexcitation is turned off, electron-hole pair production stops but the excess electrons continue to be removed by reactions with protons in the medium. The accumulated electrons do not stay on the nanoparticle indefinitely. The molar amount of thionine reduced therefore would not be a direct measure of the number of electrons extracted from the nanoparticle but would rather be a reflection of the ratio of the rate of electron transfer to thionine to that of rate of electron consumption by capture by protons. One can build an analytical model to relate these rates to the accumulated electron concentration on the nanoparticles; but treating a dynamically evolving system using a titration approach appears to be flawed. The resulting numbers are unlikely to be quantitatively representative.

Response: The concentration of proton in our neutral solution is 65 times smaller than the concentration of thionine. We added the below text on page 9 of the main text to address this concern:

"In these aqueous titration solutions, despite that the standard reduction potential of $\mathrm{Th} / \mathrm{Th}^{2-}$ is $0.064 \mathrm{~V}$ ( $v s \mathrm{NHE}$ ), the concentration of Th is 65 times higher than the proton concentration, thus the protons cannot quench significantly the accumulated electrons."

c) The accumulated electron concentration is found to be nearly the same in nanoparticles exposed to $153 \mathrm{~mW}$ of $405 \mathrm{~nm}$ light and those that were not exposed to light (see Fig. 2d). This finding may also reflect flaw/s in the thionine titration method for determination of accumulated electron concentration.

Response: As we point out in the above response, the proton concentration is quite low to significantly affect the redox titration with thionine.

d) As Figure S2 shows, the nanoparticle sample has severe heterogeneity. How does this affect the estimation of accumulated electron concentrations and batch-to-batch variations in catalytic activity? These points ought to be discussed in the main text. Statistics of nanoparticle size and shape ought to be provided as well.

Response: This is a good point to raise. The porous $\mathrm{Pd}$ nanoparticles has poor homogeneity. This is the reason why we initially thought the statistics of size and shape were not the most accurate criteria to judge the stability of the particles. Still, we added the average diameter of Pd NPs before and after photo-charging for comparison on page S3 in the SI:

"The average diameter of Pd NPs before and after photo-charging were $63.0 \pm 8.6 \mathrm{~nm}$ and $64.2 \pm$ $9.7 \mathrm{~nm}$, respectively."

As for batch-to-batch variations, we updated the below text on page 7 in the main text:

"It is important to note that the porous Pd nanoparticles have poor homogeneity (Figure S2). To account for consequent effect on the observed reaction rate constants, only two batches of synthesized nanoparticles were used. We then established the ratio of $k_{\text {Pd NPs-IPA }}$ when using the 
second batch to that standard value when using the first batch. This ratio was 1.08 and used to calibrate all the rate constants whenever using the second batch."

e) If there is significant electronic charge on Pd nanoparticles resulting simply from the use of ascorbic acid in the synthesis, as stated in the supporting information (see page S8), then the entire study ought to be re-analyzed. The effect of this residual charge and that formed by photoexcitation on the catalytic activity will need to be separated.

Response: The residual charge should be similar for each nanoparticle. Thus, the extra charge accumulated from photo-charging was resolved when changing the photo-charging condition. The comparison between the sample using photo-charged particles and the control using non-photocharged particles allows us to evaluate the catalytic contribution of photo-charging.

\section{Reviewer: 2}

Recommendation: This paper is publishable subject to minor revisions noted. Further review is not needed.

\section{Comments:}

In this manuscript, the authors reported photo-charging effect on catalysis of metallic nanoparticles. The obtained results are of some interest. However, the manuscript needs some minor revisions by considering the following points.

1. Did the charging time effect the Pd nanoparticle size? The catalytic activity of the particles dropped after a longer charging time may also be caused by the change in particle size.

Response: The Pd nanoparticles cannot grow because there is no Pd precursor used in photo-charing step. They cannot be etched by hot holes because the holes were quenched by a large amount of hole scavengers.

2. Except as a reducing agent, $\mathrm{NaBH} 4$ is also regarded as a promising hydrogen storage material, which should be introduced in the INTRODUCTION of this manuscript. Some references (such as Inorg. Chem. Front., 2021, 8,1056-1065; Inorg. Chem. Front., 2020, 7,3837-3874) can be cited.

Response: We thank the reviewer for this important point, and we added the following text and citation on page 5 of the main text. We could not add this text to the introduction due to the current flow of the manuscript.

"the reduction of 4-hydroxylaminophenol intermediate to 4-aminophenol by surface hydrogen species provided from $\mathrm{NaBH}_{4},{ }^{36,37}$,

3. There are a few typing errors spotted. The authors need to proof read the manuscript.

Response: We proof read the manuscript and made some corrections (such as amount-> number) as suggested.

\section{Reviewer: 3}

Recommendation: This paper is publishable subject to minor revisions noted. Further review is not needed.

Comments: 
This work by Pin Lyu and Son C. Nguyen reports the photo-charging effect on catalysis of metallic nanoparticles. The mechanism underlying a proportional correlation between accumulated electrons and catalytic improvement of Pd nanoparticles was proposed and was well-supported by the control experiment. This work is of considerable interest, and I recommend its publication in JPCL upon addressing my concerns as listed below:

More detailed comments:

(1) The authors reported the use of IPA as a class of hole scavengers; how to determine the degree of hole extraction in the experimental conditions?

Response: This is a good point to raise. Unfortunately, we could not measure the hole extraction directly. Instead, we estimated the number of accumulated electrons, which is indirectly correlated to the degree of hole extraction.

(2) With longer wavelength light as an excitation source (470 or $530 \mathrm{~nm}$ ), the photo-charging effect was not strong (Figure $3 \mathrm{c}$ ). How to explain this phenomenon in detail?

Response: The wavelength dependence of interband transitions was thoroughly discussed in our previous manuscript (ref 18 in the old version, ref 23 in the revised version). We provide a detailed explanation here for your reference:

Starting with the porous Pd nanoparticles used in this study, the optical absorbance of these particles in the 400-600 $\mathrm{nm}$ range (commonly studied for photocatalysis) is attributed to interband transitions because of the plasmonic coupling between adjacent crystal domains of the porous particles causes the red-shift of plasmon resonance to the near IR region. Under 405, 470, or $530 \mathrm{~nm}$ excitation, the interband transitions play a dominant role in generating the hot carriers. The shorter-wavelength excitation in the interband-transition regime generates hot electrons reside near the Fermi level, and hot holes reside deeper in the $d$-band. As we explained in the main text (page 11), the deeper holes generated from $405 \mathrm{~nm}$ excitation react easier with the hole scavenger due to its stronger oxidizing power (lower energy below the Fermi level). As expected, it results in more electrons accumulated in the nanoparticles. In contrast, the 470 or $530 \mathrm{~nm}$ excitation generates "shallower" holes, which do not have such strong oxidizing power. Hence, the nanoparticles cannot accumulate that many electrons. This trend fits to our previous work (ref 18) on the wavelength dependence of interband transitions in porous $\mathrm{Pd}$ nanoparticles, in which the quantum yield tappers off at longer wavelength.

We did not elaborate this point due to the limited length of JPC Letter. We, however, added more citations to help the readers with further clarification.

(3) For the catalysis step, the authors chose the reduction of 4-nitrophenol by $\mathrm{NaBH} 4$ as a model that demonstrated accumulated charges resulting in catalytic improvement of the photo-charged nanoparticles. Does this conclusion apply to other models?

Response: Yes, we believe the photo-charging effect may happen in many other systems and the observed catalysis may depend on specific conditions. We want to clarify that we emphasize on the background effect of photo-charging on photocatalysis more than the improvement of catalytic activity of photo-charged particles. We added the below text in the conclusion to clarify this point.

"The photo-charged metallic nanoparticles may still catalyze chemical reactions after stopping irradiation, and this process should be considered in photocatalysis." 
(4) Can the density of electrons on the surface Pd nanoparticles reach a saturated state when exciting the nanoparticles for a long time?

Response: We thank the reviewer for this good point. In principle, the Pd nanoparticles should have an upper limit of accumulated electrons. At that point, a newly generated hot hole will be quenched by one of the numerous ground electrons (but not the hole scavenger), and the newly generated hot electron will replace that consumed electron. This upper limit was also discussed in Jain's work, and it is affected by the double layer capacitance of metallic nanoparticles in water (Nano Lett. 2016, 16, 5, 3399-3407, Angew. Chem. Int. Ed. 2020, 59, 2085 -2088). 